\title{
Alterations in brain morphology by MRI in adults with neurofibromatosis 1
}

Su Wang ${ }^{1}$, Victor-Felix Mautner ${ }^{2}$, Ralph Buchert ${ }^{3}$, Stephane Flibotte ${ }^{4}$, Per Suppa ${ }^{5}$, Jan M. Friedman ${ }^{1^{*}+}$ (D) and Manraj K. S. Heran ${ }^{6+}$

\begin{abstract}
Objective: Neurofibromatosis 1 (NF1) is a rare autosomal dominant disease that causes the dysregulated growth of Schwann cells. Most reported studies of brain morphology in NF1 patients have included only children, and clinical implications of the observed changes later in life remain unclear. In this study, we used MRI to characterize brain morphology in adults with NF1.
\end{abstract}

Methods: Planar (2D) MRI measurements of 29 intracranial structures were compared in 389 adults with NF1 and 112 age- and sex-matched unaffected control subjects. The 2D measurements were correlated with volumetric (3D) brain measurements in 99 of the adults with NF1 to help interpret the 2D findings. A subset $(n=70)$ of these NF1 patients also received psychometric testing for attention deficits and IQ and was assessed for clinical severity of NF1 features and neurological problems. Correlation analysis was performed between the MRI measurements and clinical and psychometric features of these patients.

Results: Four of nine corpus callosum measurements were significantly greater in adults with NF1 than in sex-and age-matched controls. All seven brainstem measurements were significantly greater in adults with NF1 than in controls. Increased corpus callosum and brainstem 2D morphology were correlated with increased total white matter volume among the NF1 patients. No robust correlations were observed between the 2D size of these structures and clinical or neuropsychometric assessments.

Conclusion: Our findings are consistent with the hypothesis that dysregulation of brain myelin production is an important manifestation of NF1 in adults.

\section{Introduction}

Neurofibromatosis 1 (NF1), an autosomal dominant disease caused by mutations of the NF1 gene, affects approximately 1 in 3000 live births [1-3]. NF1 causes a wide range of clinical features: neurofibromas, café-aulait macules, macrocephaly, learning disabilities, and attention deficits [4-15]. Associated gliomas, malignant

\footnotetext{
*Correspondence: jan.friedman@ubc.ca

${ }^{\dagger}$ Jan M. Friedman and Manraj K. S. Heran have contributed equally to this work.

${ }^{1}$ Department of Medical Genetics, University of British Columbia,

Children's and Women's Hospital, 4500 Oak Street, Vancouver, BC V6H 3N1, Canada

Full list of author information is available at the end of the article
}

peripheral nerve sheath tumours, skeletal dysplasias, and cardiovascular disease may cause serious disability or death in affected children or young adults. The NF1 gene encodes neurofibromin, which is a negative regulator of the RAS cellular proliferation pathway [16]. Loss of NF1 function results in increased Schwann cell proliferation, a primary feature of NF1 pathology [16].

Many studies have used magnetic resonance imaging (MRI) to study brain morphology in children and adults with NF1. NF1 neuroimaging studies have been especially focused on unidentified bright objects, optic nerve/ chiasmatic expansions, and tumours of the peripheral nerves or central nervous system [17, 18]. Most people with NF1 do not develop brain tumours but may exhibit 
other, more subtle alterations of brain morphology on MRI examination, including increases in total white matter volume, total brain volume, corpus callosum (CC) area, CC length, and optic nerve tortuosity $(\mathrm{ONT})[6,7$, 9, 10, 19-28]. The clinical implications, if any, of these findings are unclear, given the small number and young age of individuals with NF1 in most reported studies [10].

Previous research has attempted to correlate brain morphological differences with cognitive or behavioural abnormalities in children with NF1, but inconsistent results have been observed $[10,24,29,30]$. To date, there have been no large-scale MRI studies of brain morphological differences and their possible relationship to cognitive or behavioural abnormalities in adults with NF1.

We characterized 2D brain morphological differences in adults with NF1 within three regions of interest: the $\mathrm{CC}$, brainstem, and optic nerves and ocular globes. We correlated the 2D measurements for the $\mathrm{CC}$ and brainstem with 3D volumetric measurements in NF1 to assess the role of different brain composition in the structural changes. Finally, we examined the associations of these 2D brain morphological changes with neuropsychometric findings among adults with NF1.

\section{Methods}

\section{Participants}

Between 2003 and 2015, all patients with a clinical diagnosis of NF1 seen in the NF Outpatient Department of the University Hospital Hamburg-Eppendorf in Hamburg, Germany, were offered brain MRI to monitor their intracranial tumour burden. Head MRI was obtained on 434 adults with NF1; each patient was imaged on three separate occasions, on average. Participants who were found to have optic gliomas or brain tumors were excluded from the current study. Sex and age were recorded at the time of each examination.

Unaffected control individuals had undergone head MRI at Vancouver General Hospital, Vancouver, Canada, for headaches or non-specific neurologic symptoms or signs suggestive of a mass lesion. Controls were age(within 24 months) and sex-matched to $25 \%$ of the NF1 cohort chosen at random. Affected and unaffected participants were excluded if no MRI image was available that permitted clearly-defined morphological measurements. In total, 389 adults with NF1 and 112 unaffected controls were included in the morphological analysis. In cases where multiple sets of MRI scans were obtained for one individual, the most recent MRI scan was used for brain morphological measurements.

A subset of adults with NF1 $(n=70)$ received clinical evaluations for NF1 severity, neurological severity, attention deficit hyperactivity disorder (ADHD) severity, and neuropsychometric testing for attention deficiencies and intelligence quotient (IQ).

\section{Morphological measurements}

Head MRI exams were conducted in a 1.5 or 3.0 Tesla scanner. Multiple coronal, axial, and/or sagittal T1, T2, and FLAIR images were obtained. OsiriX Lite [31] software was used to obtain measurements in NF1 patients, and IMPAX PACS (Agfa, Ridgefield Park, NJ) software was used for this purpose in unaffected controls. Image slices were $\leq 5.5 \mathrm{~mm}$ thick for adults with NF1 and $\leq 6 \mathrm{~mm}$ thick for unaffected populations. In total, 29 measurements were obtained for each MRI set for each participant.

Eight measurements were obtained for the CC: area, length, height, genu width, anterior body width, midbody width, posterior body width, and splenium width (Fig. 1A). CC height was measured as maximum height of CC perpendicular to a line drawn at base of the CC.

Seven measurements were obtained for each of the ocular globes: anterior to posterior (AP) length, diameter, anterior to diameter $(\mathrm{AD})$ length, anterior to interzygomatic line (AIZ) length, posterior to interzygomatic line (PIZ) length, optic nerve displacement (OND), and optic nerve path (ONP) (Fig. 2A). AP and diameter measurements were obtained at maximum length/width. $\mathrm{AD}$ was measured as maximum length perpendicular to diameter. AIZ and PIZ was measured as maximum length perpendicular to interzygomatic line.

Seven measurements were obtained for the brainstem: midbrain width, midbrain AP length, pons AP length, left and right middle cerebellar peduncle (MCP) lengths, medulla oblongata width, and medulla oblongata AP length (Fig. 3A). Midbrain width, pons AP, left and right $\mathrm{MCP}$, and medulla oblongata width were measured as maximum length/width. Midbrain AP and medulla oblongata AP length were measured as maximum length perpendicular to midbrain width and medulla oblongata width, respectively.

$\mathrm{CC}$ bulbosity was calculated by dividing the splenium width by the posterior body width. Ocular globe elongation was calculated by dividing AD length by AP length. Globe protrusion was calculated by dividing AIZ by the sum of AIZ and PIZ. Optic nerve tortuosity (ONT) was calculated by dividing ONP by OND.

Brain volumes were measured according to methods described in our companion paper [28].

\section{Clinical and neuropsychometric assessments Clinical NF1 severity}

All NF1 patients were evaluated clinically by Dr. Victor Mautner. NF1 clinical severity was categorised by Dr. Mautner using a modified Riccardi scale [32, 33]. 


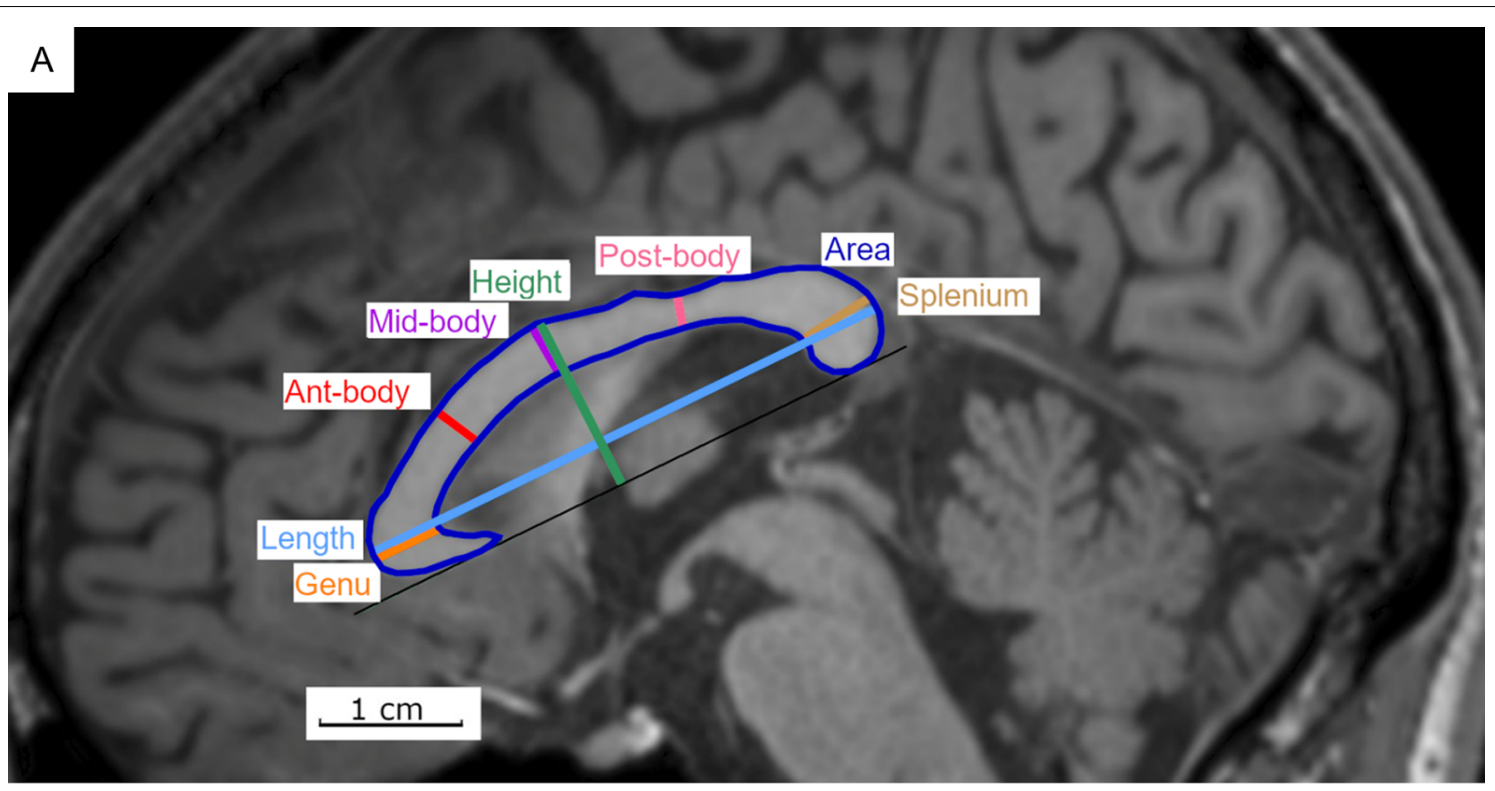

B

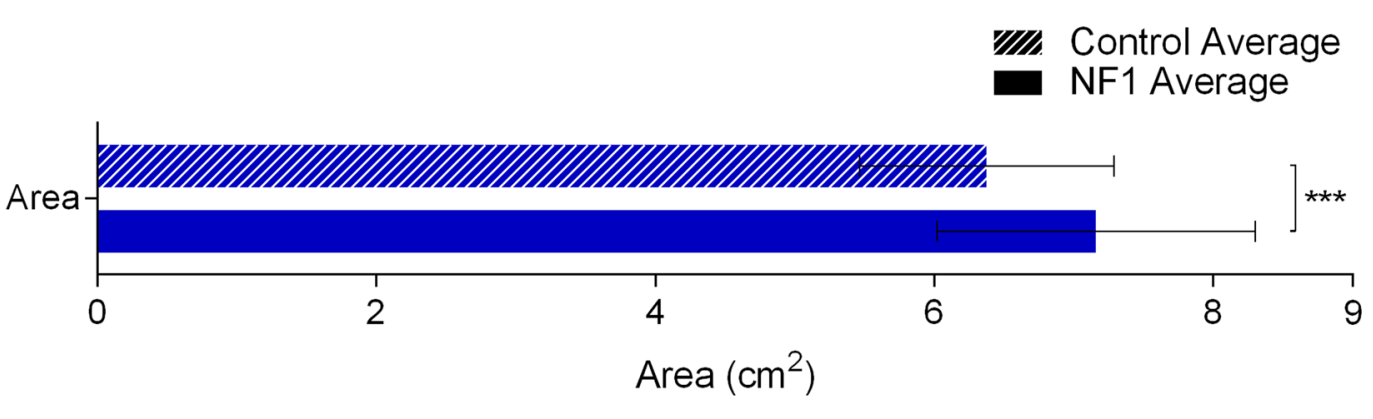

C

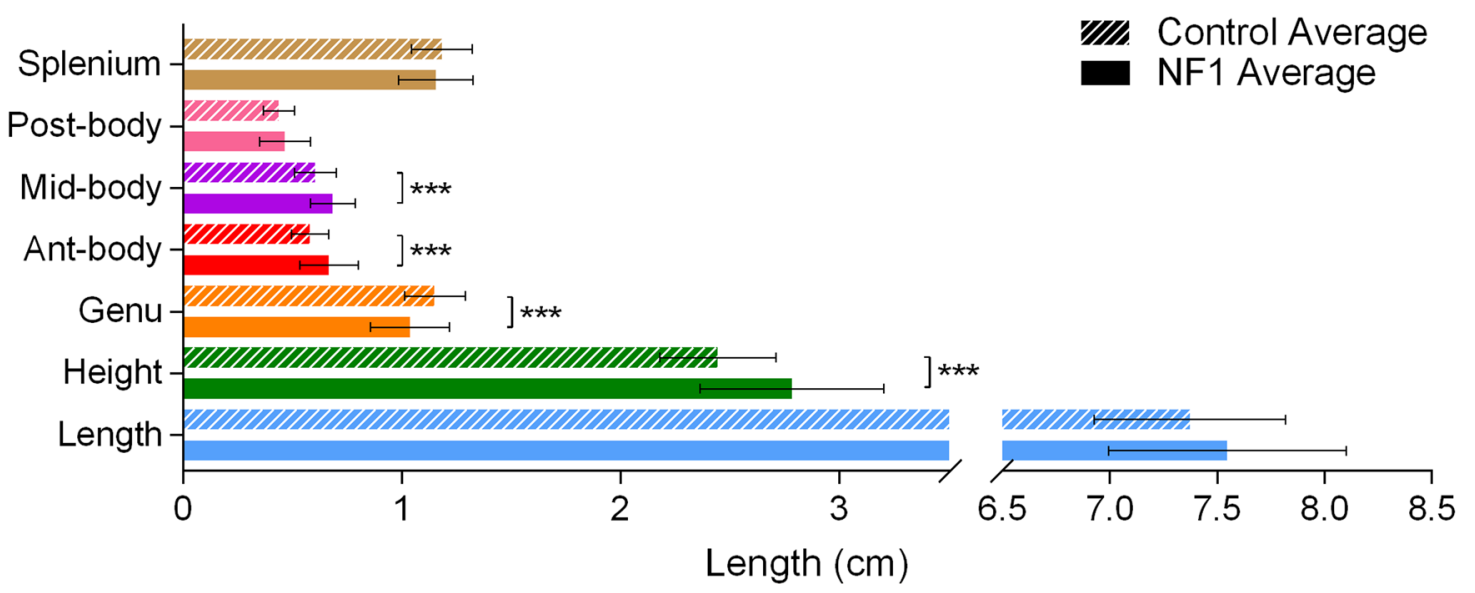

Fig. 1 Corpus callosum measurements and averages for adults with NF1 and unaffected control participants. A Representative MRI image showing T1-weighted midline sagittal view of a 33-year-old male with NF1. B Average CC midsagittal area. C Average CC measurements. Coloured lines and area outlined in $\mathbf{A}$ show where structures indicated by the same colour in $\mathbf{B}$ or $\mathbf{C}$ are measured. Error bars indicating one standard deviation and asterisks indicating FDR-adjusted statistical significance are shown in $\mathbf{B}$ and $\mathbf{C} . p<0.001={ }^{* * *}$. Abbreviations used in figure: Genu $=$ genu width, Ant-body= anterior body width, Mid-body= mid-body width, Post-body= posterior body width, and Splenium = splenium width 


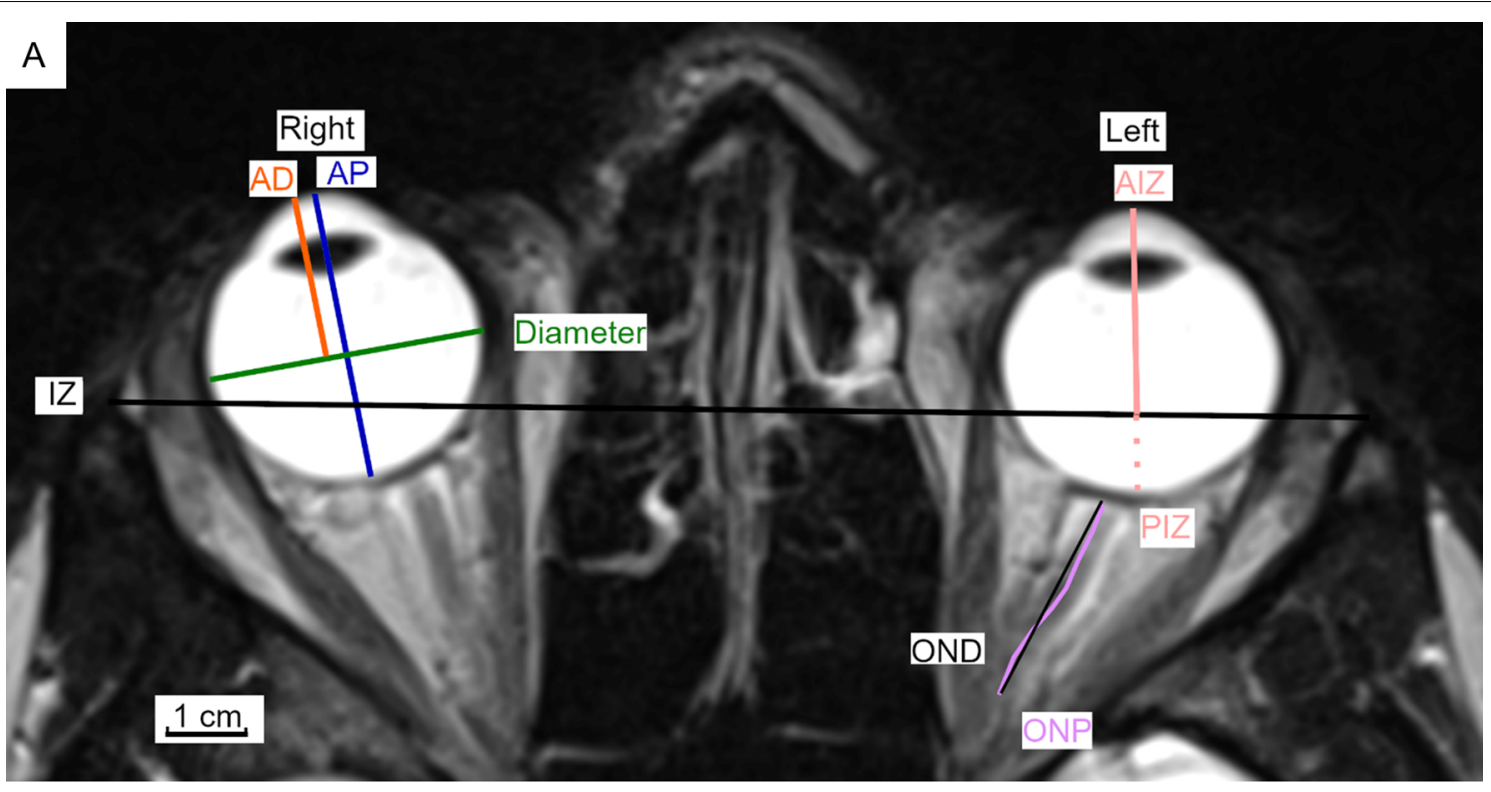

B

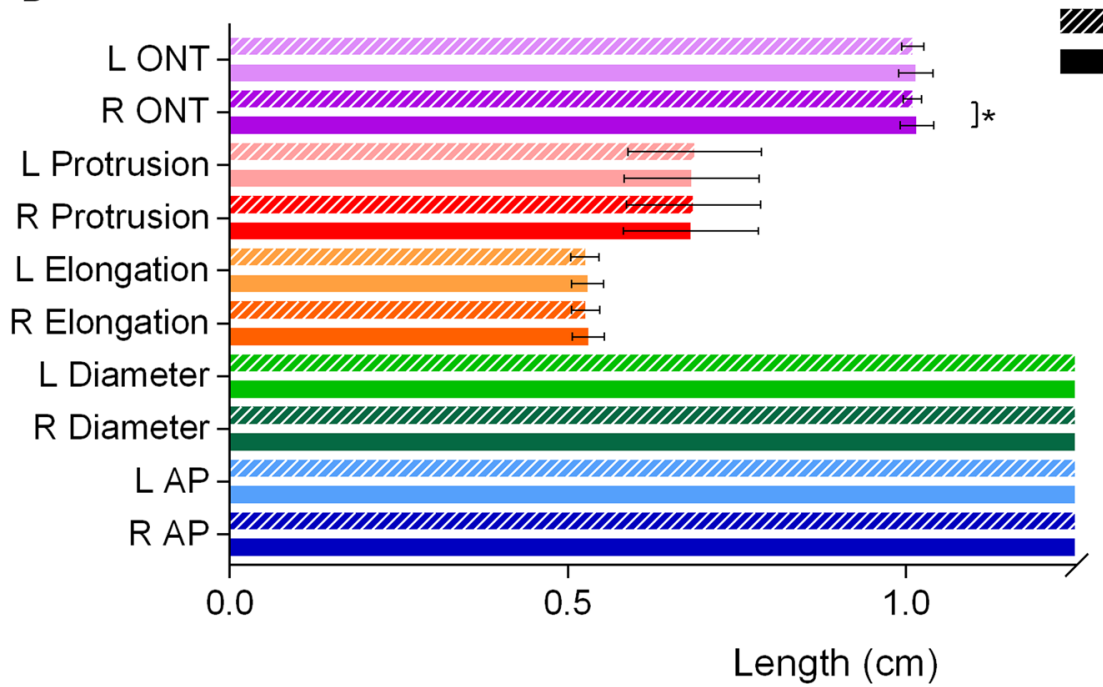

WII. Control Average

- NF1 Average

Length $(\mathrm{cm})$

Fig. 2 Ocular globe measurements and averages for adults with NF1 and unaffected control participants. A Representative MRI image showing T2-weighted axial view of a 25-year-old male with NF1. B Select average ocular globe measurements. Unique measurements are shown on one side but were obtained on both left and right side. Coloured lines in $\mathbf{A}$ show where structures indicated by the same colour in $\mathbf{B}$ are measured. Error bars indicating one standard deviation and asterisks indicating FDR-adjusted statistical significance are shown in $\mathbf{B} . p<0.5={ }^{*}$; $p<0.001={ }^{* * *}$. Abbreviations used in the figure: $L=$ left, $R=$ right, $A P=$ anterior to posterior length, $A D=$ anterior to diameter length, $A \mathrm{IZ}=$ anterior to interzygomatic line length, $\mathrm{IZ}=$ interzygomatic line, $\mathrm{PIZ}=$ posterior to interzygomatic line length, $\mathrm{OND}=$ optic nerve displacement, and $\mathrm{ONP}=$ optic nerve path

Intellectual and psychological functions were excluded from the Riccardi scale to avoid confounding between learning disability and medical severity in the correlation analysis.

\section{Clinical neurological severity}

Clinical neurological severity for each NF1 patient was categorised by Dr. Mautner into one of four grades: Patients were classified as Grade 1 if they had no 

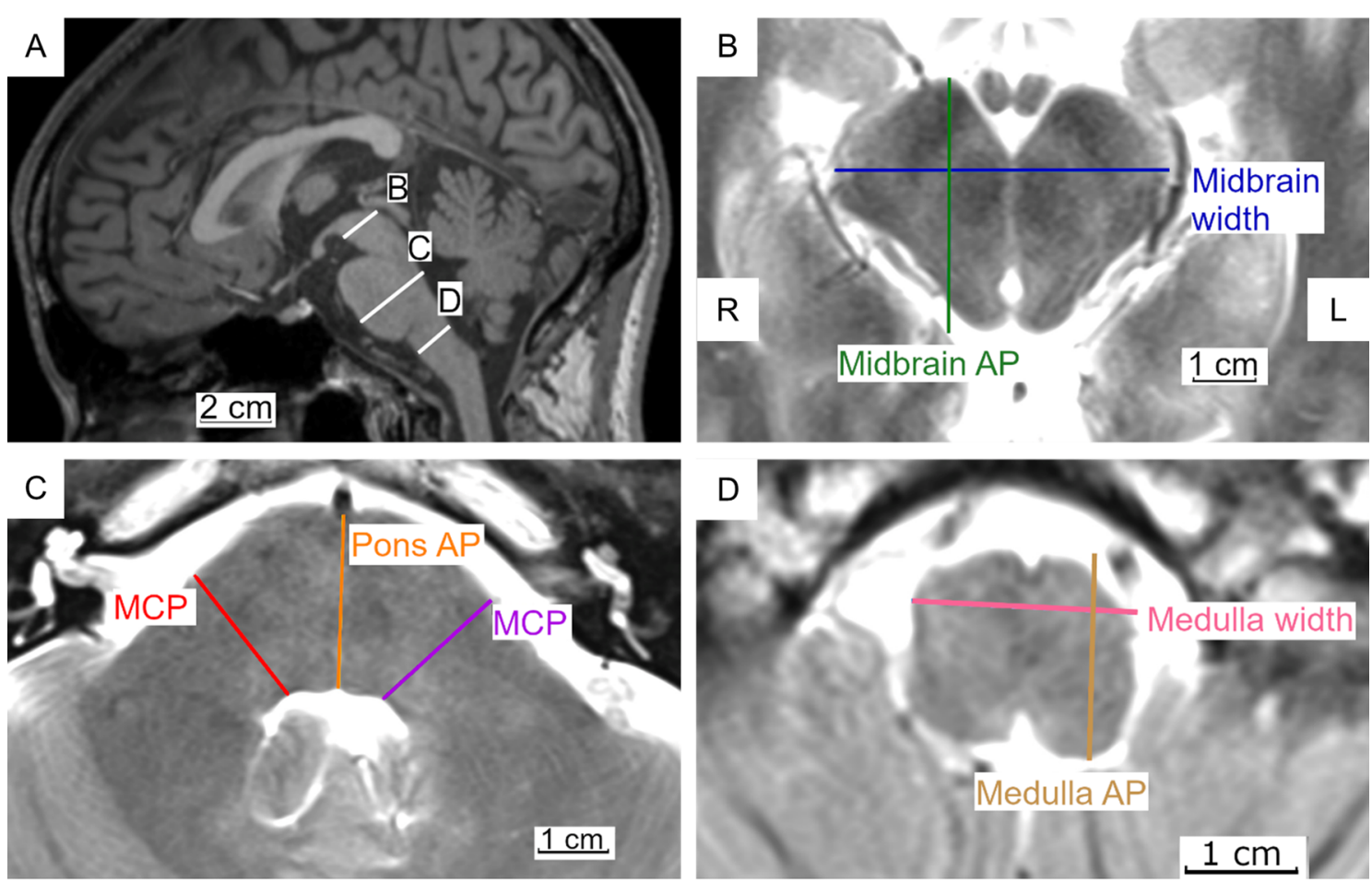

E

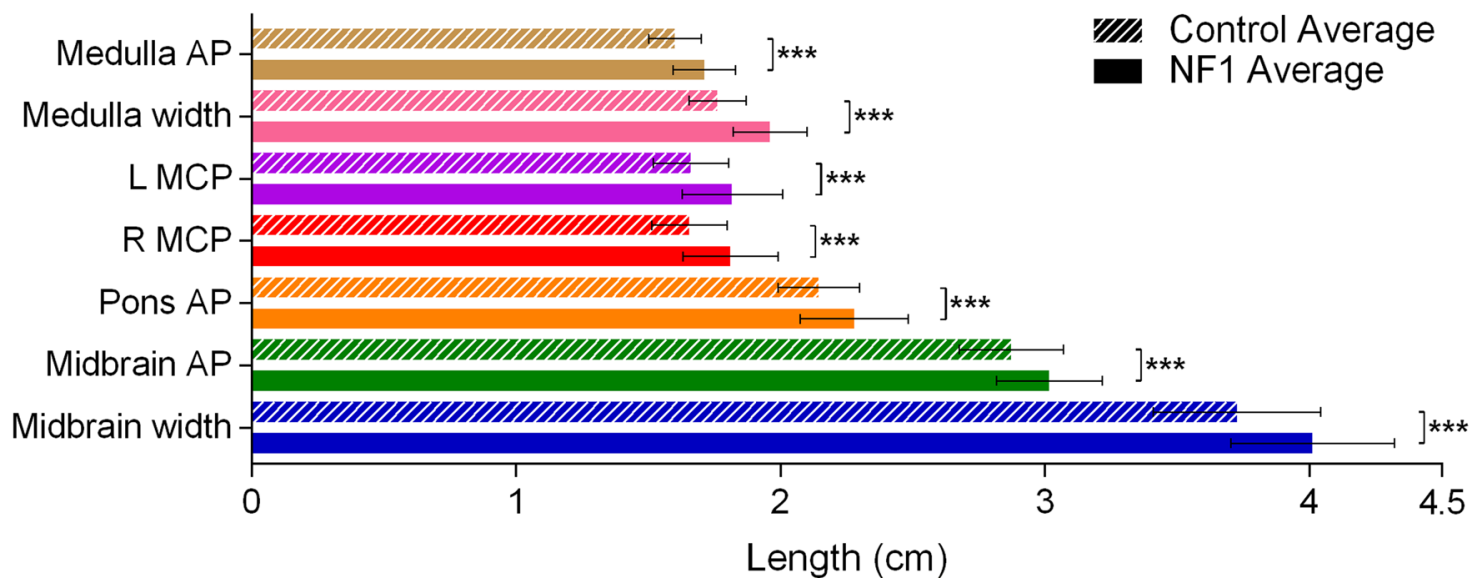

Fig. 3 Location of brainstem measurements shown on representative sagittal (A), axial (B), (C), and (D) images, and average measurements (E) for adults with NF1 and unaffected control participants. A-D Representative MRI images showing T1-weighted midline sagittal and T2-weighted axial views of a 25-year-old male with NF1. E Average brainstem measurements. The labeled lines in $\mathbf{A}$ show position of the midbrain (B), the pons and the middle cerebellar peduncle (C), and the medulla oblongata (D). Coloured lines in B-D show where structures indicated by the same colour in $\mathbf{E}$ are measured. Error bars indicating one standard deviation and asterisks indicating FDR-adjusted statistical significance are shown in $\mathbf{E}$. $p<0.001=* * *$. Abbreviations used in the figure: $\mathrm{L}=$ left, $\mathrm{R}=$ right, $\mathrm{AP}=$ anterior to posterior length, and $\mathrm{MCP}=$ middle cerebellar peduncle

neurological deficits. Grade 2 patients included those with discrete neurological deficits only-muscle hypotonia, sensation deficits, balance problems, or speech problems. Grade 3 included patients more clear neurological dysfunction-paresis, ataxia, significant ocular motor deficits, or substantial pain. Grade 4 encompassed patients with neurological deficits that seriously compromised health-intractable pain, paralysis, or treatment-resistant seizures.

\section{Clinical attention deficit hyperactivity disorder (ADHD) severity}

Clinical ADHD severity for adults with NF1was categorized into three grades using the established DSM-IV 
criteria [34]. Grade 1 encompassed no clinical diagnosis. Grade 2 was subclinical attention deficit with no ADHD diagnosis. Grade 3 included those with a diagnosis of either attention deficit disorder (ADD) or ADHD.

\section{Intelligence quotient (IQ)}

Full-scale, verbal, and performance IQ were obtained using the Wechsler adult intelligence scale-revised (WAIS-R) [35]. WAIS-R test scores were standardized according to age and sex.

\section{Attention deficit measurement}

Attention deficit measurements were obtained using the Visual Test of Variables of Attention, version 7.0.3 or 8.0 [36]. Measurements were obtained for variability of response time (consistency), response time, commission error (impulsivity), errors of omission (inattention), post-commission response times, and multiple and anticipatory responses. An Attention Comparison Score was calculated for each patient. All test scores were standardized according to age and sex.

\section{Statistical analysis}

All data were analysed using $\mathrm{R}$ Studio 3.4.1 [37]. A false discovery rate (FDR)-adjusted significance level of $p<0.05$ was used throughout to account for multiple comparisons [38].

\section{Demographic analysis}

Mean age was compared between adults with NF1 and unaffected controls using the Student $\mathrm{T}$ test after demonstrating satisfactory dataset normality and variance using the Shapiro-Wilk test and Fisher's F test, respectively. Sex ratios were compared between adults with NF1 and unaffected controls using the $\chi^{2}$ test.

\section{Brain morphological analysis}

Means of the 26 different brain morphological measurements were compared between adults with NF1 and unaffected controls. Student-T tests were used for normally distributed data. Non-normal distributions were compared using Mann-Whitney U tests. CC bulbosity means were compared between males and females for both adults with NF1 and unaffected adults using twoway ANOVA test. Dataset normality and variance were determined using the Shapiro-Wilk test and Fisher's F test respectively. Significance ( $p$ value) was adjusted using FDR to account for 26 comparisons.

\section{Brain morphological: volumetric correlation analysis}

Brain morphological-volumetric correlation analysis was conducted for NF1 patients with a complete set of 2D CC or brainstem measurements and 3D volumetric measurements. Principal component analysis (PCA) was calculated for $\mathrm{CC}$ and brainstem separately. Principal components were included based on a combination of eigenvalue (greater than 1) and minimum combined amount of variance it accounts for (70\%). Pearson correlation was conducted, and $\mathrm{p}$ values were adjusted using FDR to account for multiple comparisons.

\section{Brain morphology-neuropsychometric correlation analysis} Brain morphology measurements for the CC, ocular globes, and brainstem were independently grouped to maximize the number of subjects included in the PCA for each region of interest; the PCA included only individuals with a complete set of measurements for each region. Principal components were selected for the correlation analysis if their eigenvalue was greater than 1 and their combined variance accounted for at least $70 \%$ of the total. Clinical NF1 severity, clinical neurological severity, and clinical ADHD diagnosis were analysed as ordinal data. Total IQ from the WAIS-R and Attention Comparison Score were analysed as continuous measurements. Principal components from the three brain structures measured were independently compared to the neuropsychometric measurements using Pearson correlation for continuous data and Spearman's Rank-Order correlation for ordinal data. No significance testing was conducted on the correlation matrix due to insufficient sample size.

\section{Results}

\section{Demographics}

This study included 494 participants: 389 adults with NF1 and 112 age- (within 24 months) and sex-matched unaffected controls. There were no statistically significant differences in age or sex ratio distribution between adults with NF1 and unaffected controls overall or in the subgroups in whom the three brain structures $(\mathrm{CC}$, ocular globes, and brainstem) were measured by MRI (Table 1 ).

\section{Brain morphology comparison between NF1 patients and controls}

Four of eight corpus callosum measurements (midsagittal length, height, anterior body width, mid body width) were significantly greater and one (genu width) was significantly shorter in adults with NF1 than in unaffected control participants (Fig. 1B, C). CC length, posterior body width, splenium width, and $\mathrm{CC}$ bulbosity did not differ between the two groups. The statistical significance was determined after adjustment for multiple comparisons.

Of the five measurements made for each ocular globe, only the AP lengths were significantly reduced in both eyes in adults with NF1 compared to unaffected controls 
Table 1 Participant demographics for adults with NF1 and matched unaffected controls

\begin{tabular}{|c|c|c|}
\hline & Adults with NF1 & Unaffected controls \\
\hline \multicolumn{3}{|l|}{ All subjects } \\
\hline N & 389 & 112 \\
\hline Mean age (SD) & $37.4(13.5)$ & $39.4(13.1)$ \\
\hline Age range & $18.1-72.9$ & 19.4-73.3 \\
\hline $\begin{array}{l}\text { Female: Male } \\
\text { (Female Percentage) }\end{array}$ & $218: 171(56.0 \%)$ & $63: 49(56.2 \%)$ \\
\hline \multicolumn{3}{|c|}{ Subjects with one or more MRI measurements of corpus callosum } \\
\hline $\mathrm{N}$ & 226 & 57 \\
\hline Mean age (SD) & $37.1(13.6)$ & $38.0(13.2)$ \\
\hline Age range & $18.2-72.9$ & $19.4-64.7$ \\
\hline $\begin{array}{l}\text { Female: male } \\
\text { (female percentage) }\end{array}$ & $126: 100(55.7 \%)$ & $31: 26(54.4 \%)$ \\
\hline \multicolumn{3}{|c|}{ Subjects with one or more MRI measurements of ocular globes } \\
\hline N & 386 & 96 \\
\hline Mean age (SD) & $37.4(13.5)$ & $39.6(13.7)$ \\
\hline Age range & $18.1-72.9$ & $19.4-73.3$ \\
\hline $\begin{array}{l}\text { Female: male } \\
\text { (female percentage) }\end{array}$ & $214: 172(55.4 \%)$ & $56: 40(58.3 \%)$ \\
\hline \multicolumn{3}{|c|}{ Subjects with one or more MRI measurements of brain stem } \\
\hline N & 375 & 96 \\
\hline Mean age (SD) & $37.2(13.3)$ & $39.5(13.1)$ \\
\hline Age range & $18.1-72.9$ & 19.4-73.3 \\
\hline $\begin{array}{l}\text { Female: male } \\
\text { (female percentage) }\end{array}$ & 209:166 (55.7\%) & $56: 40(58.3 \%)$ \\
\hline
\end{tabular}

(Fig. 2B). Optic nerve tortuosity was greater among adults with NF1 than among unaffected individuals. This difference was small and reached statistical significance only on the left side.

The brainstems of individuals with NF1 were significantly larger than expected in all seven sites measured (midbrain width, midbrain AP length, pons AP length, left and right MCP lengths, medulla oblongata width, and medulla oblongata AP length) (Fig. 3E).

\section{Brain morphology: white matter composition correlation in patients with NF1}

2D morphological measurements do not distinguish between grey or white matter composition of the structures measured. In order to determine whether the increased $\mathrm{CC}$ and brainstem measurements we observed were related to altered myelination (white matter), we correlated $2 \mathrm{D}$ brain morphological measurements with $3 \mathrm{D}$ brain volumetric measurements in individuals with NF1. Principal component analysis was used to identify combinations of $\mathrm{CC}$ or brainstem 2D measurements that distinguished NF1 patients. Ninety-nine adults with NF1 (47 females and 52 males) had complete $2 \mathrm{D}$ and $3 \mathrm{D}$ measurements available for the $\mathrm{CC}$. The first principal component $(\mathrm{PC} 1)$ reflects a combination of $\mathrm{CC}$ area and mid-body measurements so that a larger PC1 indicates greater CC area and midbody width. Larger PC2 indicates shorter and flatter CC. Larger PC3 indicates less bulbous CC posterior body. Larger PC4 indicates male. Overall, we found that larger $2 \mathrm{D}$ corpus callosum measurements most strongly correlated with increased total brain volume and increased corpus callosum white matter volume (Fig. 4A).

There were 55 individuals with NF1 (25 females and 30 males) who had complete 2D and 3D measurements for the brainstem. The top three principal components based on 2D brainstem measurements were selected for correlation analysis. Larger PC1 indicates smaller pons and MCP, larger PC2 indicates older age, and larger PC3 indicates female. We found that larger 2D pons and MCO measurements most strongly correlate with increased total brain volume and total white matter volume (Fig. 4B).

These results suggest that the greater than expected $2 \mathrm{D}$ corpus callosum and brainstem measurements reflect 

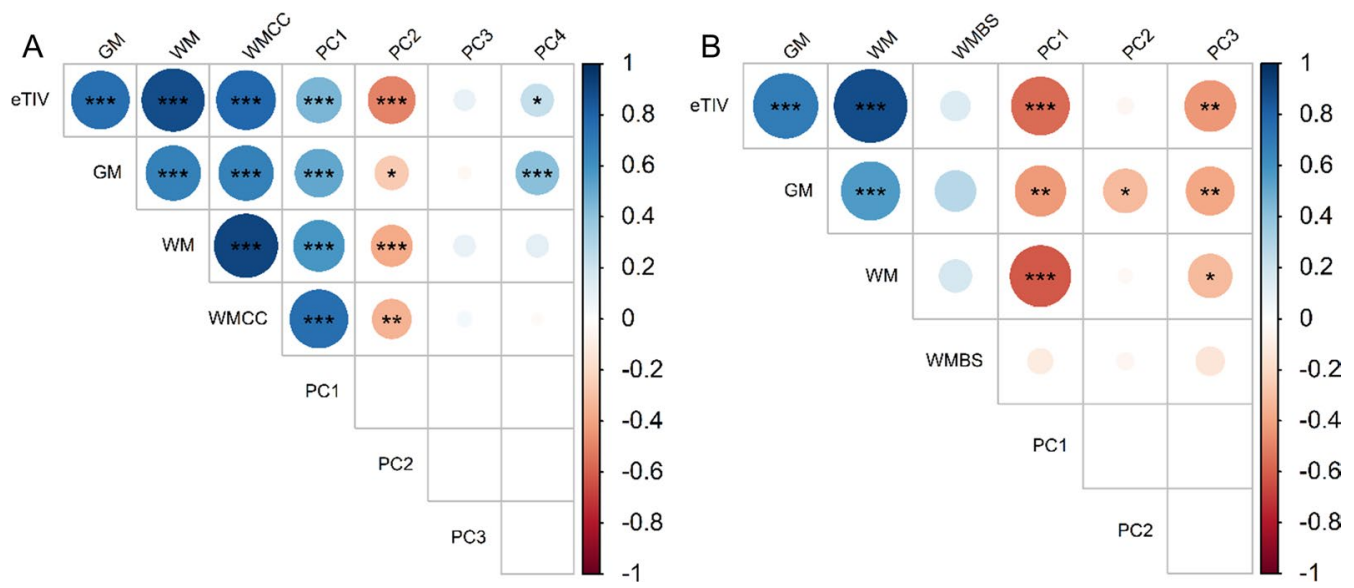

Fig. 4 Correlation matrix of principal components of 2D brain morphological measurements with 3D brain volumetric measurements of the corpus callosum (A) and brainstem (B) in adults with NF1.The color scale represents the degree of correlation from strongly negative $(-1$, red) to strongly positive $(1$, blue). The size of the circles also indicates the strength of the correlation, with values closer to -1 or 1 larger than those that are closer to 0 . Asterisks indicate FDR-adjusted statistical significance. $p<0.05={ }^{*}, p<0.01={ }^{* *}, p<0.001={ }^{* * *}$. Abbreviations: eTIV $=$ total brain volume, $\mathrm{GM}=$ grey matter volume, $\mathrm{WM}=$ white matter volume, $\mathrm{WMCC}=$ corpus callosum white matter volume, $\mathrm{WMBS}=$ brainstem white matter volume, and $\mathrm{PC}=$ principal component

increased total white matter volume, and, by extension, increased myelination.

\section{Morphology: neuropsychometric correlations}

The average IQ for patients in the NF1 group was 107.4 $(\mathrm{SD}=17.3)$ for those with complete corpus callosum measurements, 100.0 (SD=19.1) for patients with complete ocular globe measurements, and 101.0 ( $\mathrm{SD}=21.8)$ for patients with complete brainstem measurements.

Twenty-nine adults with NF1 had complete datasets for the clinical and neuropsychometric assessments as well as for the CC measurements analysed. The top three principal components were selected for the CC. None of the CC principal components was strongly correlated with any of the five clinical assessments or neuropsychometric measurements (Table 2).

Twenty-six adults with NF1 had complete datasets for the clinical and neuropsychometric assessments and brainstem measurements analysed. The top three principal components were selected for the brainstem. PC1 was correlated with both decreased neurological severity $\left(r_{s}=-0.51\right)$ and negative clinical ADD/ADHD diagnosis $\left(r_{s}=-0.49\right)$. The three largest weights for PC1 were the right MCP length $(-0.42)$, left MCP length $(-0.41)$, and the pons AP length $(-0.40)$. Thus, a smaller PC1 value indicates an overall thicker middle brainstem. Clinical severity was correlated with decreased clinical ADD/ ADHD diagnosis $\left(r_{s}=-0.56\right)$, and neurological severity was correlated with decreased IQ $\left(r_{s}=-0.38\right)$ (Table 3$)$.

Table 2 Correlation values and $p$ values of corpus callosum measurements with clinical and neuropsychometric assessments in adults with NF1

\begin{tabular}{|c|c|c|c|c|c|c|c|}
\hline & Neuro. sev & ADD/ADHD & IQ & ACS & PC1 & PC2 & PC3 \\
\hline Clin.sev & 0.14 & -0.49 & -0.29 & -0.17 & 0.38 & -0.05 & -0.02 \\
\hline Neuro. sev & & 0.55 & 0.20 & -0.41 & 0.19 & -0.05 & 0.17 \\
\hline ADD/ADHD & & & 0.26 & -0.17 & 0.01 & -0.20 & -0.06 \\
\hline IQ & & & & 0.10 & -0.01 & -0.41 & 0.33 \\
\hline ACS & & & & & -0.22 & 0.23 & 0.08 \\
\hline PC1 & & & & & & -0.20 & -0.03 \\
\hline$P C 2$ & & & & & & & -0.09 \\
\hline
\end{tabular}

Top three corpus callosum PCs are correlated to neuropsychometric measurements (clinical severity, $n=70$; clinical neurological severity, $n=70$; clinical ADD/ ADHD diagnosis, $n=50 ; I Q, n=61$; and Attention Comparison Score, $n=68$ ). The correlation matrix combines the Pearson correlation and Spearman correlation (italicized) results. Abbreviations used in the table: Clin. Sev.=clinical NF1 severity, Neuro. Sev. =clinical neurological severity, ADD/ADHD =clinical Attention deficit hyperactivity disorder severity, IQ = intelligence quotient, $\mathrm{ACS}=$ Attention Comparison Score, and $\mathrm{PC}=$ principal component 
Table 3 Correlation values and p-values of brainstem measurements with clinical and neuropsychometric assessments in adults with NF1

\begin{tabular}{|c|c|c|c|c|c|c|c|}
\hline & Neuro. sev & ADD/ADHD & IQ & ACS & PC1 & PC2 & PC3 \\
\hline Clin.sev & -0.06 & -0.56 & -0.24 & -0.13 & 0.21 & -0.17 & 0.21 \\
\hline Neuro.sev & & 0.43 & -0.38 & -0.21 & -0.51 & -0.16 & -0.20 \\
\hline ADD/ADHD & & & 0.24 & -0.06 & -0.49 & 0.32 & -0.31 \\
\hline IQ & & & & 0.35 & -0.17 & 0.33 & 0.17 \\
\hline ACS & & & & & 0.05 & 0.27 & 0.30 \\
\hline PC1 & & & & & & 0.01 & 0.18 \\
\hline PC2 & & & & & & & 0.22 \\
\hline
\end{tabular}

Top three brainstem PCs are correlated to neuropsychometric measurements (clinical severity, $\mathrm{n}=70$; clinical neurological severity, $\mathrm{n}=70$; clinical $\mathrm{ADD} / \mathrm{ADHD}$ diagnosis, $n=50 ; I Q, n=61$; and Attention Comparison Score, $n=68$ ). The correlation matrix combines the Pearson correlation and Spearman correlation (italicized) results. Abbreviations used in the table: Clin. Sev. = clinical NF1 severity, Neuro. Sev. = clinical neurological severity, ADD/ADHD $=$ clinical Attention deficit hyperactivity disorder severity, $\mathrm{IQ}=$ intelligence quotient, $\mathrm{ACS}=$ Attention Comparison $\mathrm{Score}$, and $\mathrm{PC}=$ principal component

Forty-four adults with NF1 had complete datasets for the clinical and neuropsychometric assessments and ocular globe measurements analysed. The top five principal components were selected for the ocular globes. None of the variables was strongly correlated with the others (Table 4).

\section{Discussion}

We conducted the first large-scale MRI study of brain morphological differences and their relationship to cognitive or behavioural abnormalities in adults with NF1. We found that adults with NF1 have apparent enlargement of the corpus callosum and brainstem in comparison to unaffected adults. These $2 \mathrm{D}$ morphological enlargements are correlated to increased total white matter volume. In a companion study focused on brain volume, we also found an increase in total and regional white matter in the brains of adults with NF1 compared to control adults [28]. In the current study, we did not find any obvious correlation between the brain morphological changes we observed and the clinical or neuropsychometric assessments in these individuals.

In the present study, we observed increased $\mathrm{CC}$ area, height, and anterior body and mid-body widths among adults with NF1. Our findings are consistent with those of previous studies in children and smaller groups of adults with NF1 [23, 25]. Also, previous brain MRI studies in people with NF1 have found evidence of increased total white matter volume, increased brain volume, and megalencephaly $[6,7,9,10,19-22]$. Similarly, we found strong correlation between $2 \mathrm{D} \mathrm{CC}$ size and total and CC white matter volume.

Loss of function mutations of NF1 cause dysregulated proliferation in Schwann cells, which are responsible for the myelination of axons in the peripheral nervous system [16]. In the brain, white matter is mainly comprised of myelinated axons [39]. It is hypothesized that the increases in total white matter volume, total brain

Table 4 Correlation values and $p$ values of ocular globe measurements with clinical and neuropsychometric assessments in adults with NF1

\begin{tabular}{|c|c|c|c|c|c|c|c|c|c|c|}
\hline & Clin. sev & Neuro. sev & ADD/ADHD & IQ & ACS & PC1 & PC2 & PC3 & PC4 & PC5 \\
\hline & \multicolumn{10}{|c|}{ Correlation matrix } \\
\hline Clin.sev & & 0.23 & -0.21 & -0.21 & -0.21 & -0.36 & 0.01 & 0.04 & -0.40 & -0.20 \\
\hline Neuro.sev & & & 0.49 & -0.34 & -0.13 & -0.25 & 0.20 & -0.08 & 0.23 & 0.11 \\
\hline ADD/ADHD & & & & 0.04 & -0.03 & 0.13 & 0.36 & -0.18 & 0.10 & 0.26 \\
\hline IQ & & & & & 0.16 & 0.50 & -0.11 & -0.01 & -0.02 & -0.36 \\
\hline ACS & & & & & & -0.10 & -0.18 & -0.07 & 0.09 & 0.01 \\
\hline PC1 & & & & & & & 0.12 & 0.06 & 0.08 & -0.05 \\
\hline PC2 & & & & & & & & 0.06 & -0.04 & 0.04 \\
\hline PC3 & & & & & & & & & -0.05 & 0.04 \\
\hline PC4 & & & & & & & & & & 0.00 \\
\hline
\end{tabular}

Top five corpus callosum PCs are correlated to neuropsychometric measurements (clinical severity, $n=70$; clinical neurological severity, $n=70$; clinical ADD/ ADHD diagnosis, $n=50 ; I Q, n=61$; and Attention Comparison Score, $n=68$ ). The correlation matrix combines the Pearson correlation and Spearman correlation (italicized) results. Abbreviations used in the table: Clin. Sev. =clinical NF1 severity, Neuro. Sev. = clinical neurological severity, ADD/ADHD=clinical Attention deficit hyperactivity disorder severity, $\mathrm{IQ}=$ intelligence quotient, $\mathrm{ACS}=$ Attention Comparison Score, and $\mathrm{PC}=$ principal component 
volume, and megalencephaly that occur in NF1 are related to the dysregulation of oligodendrocytes, the myelin-producing cells in the central nervous system [23, 40]. If this interpretation is correct, enlargement of the $\mathrm{CC}$, a structure largely composed of white matter, might be expected in people with NF1. The decrease in CC genu width and increase in the anterior and mid-body widths raises the possibility that the $\mathrm{CC}$ shape is also changed. Enlargement of the CC height and area without significant alteration of the length is consistent with a change in $\mathrm{CC}$ shape and volume.

The enlargement of the brainstem that we observed among adults with NF1 is a novel finding. The brainstem is comprised of both grey and white matter, with the MCP (significantly enlarged among adults with NF1) comprised mostly of white matter [41, 42]. This is further evident by the strong correlation we found between increased brainstem 2D morphology measurements (specifically pons and $\mathrm{MCP}$ ) and increased total white matter volume. Thus, the brainstem enlargement we observed is also consistent with dysregulated myelin proliferation in individuals with NF1.

Both age and sex are known to affect CC size, ocular globe size and position, and brainstem size [43-47]. We carefully matched unaffected individuals by age and sex to avoid confounding by these factors in our analysis. However, the adult NF1 group was recruited from Hamburg, Germany, while the unaffected comparison group was obtained in Vancouver, Canada. Different MR imaging procedures and measurement software were used for the NF1 group and the unaffected comparison control group. As well, the NF1 and comparison groups probably differed in ancestry, and some differences in brain morphology have been associated with ethnicity [48-50]. However, all of the statistically significant differences in brain morphological measurements that we observed between NF1 and control subjects were relatively large $(>0.05 \mathrm{~cm})$, and the differences in white matter volume found in our companion paper were in comparison to a control group studied in Hamburg using the same MRI scanners as these NF1 patients [28].

Another limitation of our study is the lack of dedicated orbital MRIs. The asymmetry in the ONT we observed probably does not represent a true anatomical difference and may be a result of measurement error, as previous studies have not found ONT asymmetry [26, 27]. The current study is less accurate than Ji et al.s [27], as we only measured ONT in one axial plane while Ji and associates used dedicated 3-dimensional magnetization-prepared rapid gradient echo sequences with $1 \mathrm{~mm}$ slices.

Our study specifically excluded NF1 patients with optic pathway gliomas. Optic pathway gliomas are present in $15-20 \%$ of children with NF1, and, although the prevalence is lower in adulthood, some of these tumors do persist [51]. We do not know if the presence of an optic pathway glioma affects other measurements of the ocular globes or extraocular brain structures, and excluding patients with such tumors may have affected our results.

Our correlation analyses between structures measured on MRI and clinical/neuropsychometric assessments are limited by small sample sizes as only a subset of the adults with NF1 had psychometric testing. The average IQ of NF1 patients in our study was similar to that expected in the general population; this finding differs from previous studies which found the average IQ of individuals with NF1 to be about 1 standard deviation below that of unaffected subjects [52]. Some previous studies also found that increased CC volume or CC index correlated with decreased academic achievement and IQ in children with NF1 [10, 24], although a more recent study using diffusion tensor imaging to examine myelination of white matter specifically failed to find a significant relationship between total CC area and IQ scores in children with NF1 [30]. Kayl et al. [29] found that among children with NF1, smaller splenium size was correlated with increased attention problems as reported by teachers. We did not find any robust correlations between the brain morphology and neuropsychometric measurements in adults with NF1.

\section{Conclusion}

We conducted the largest study of 2D brain morphology in adults with NF1 reported to date to characterize the brain morphology alterations. The enlargement of the CC and brainstem and correlation to increased total white matter volume that we observed in adults with NF1 lends support to the hypothesis that neurofibromin haploinsufficiency causes dysregulation of myelin production in the brain. The relationship of this overgrowth of myelinated brain structures to the frequent occurrence of central nervous system gliomas and of benign and malignant peripheral nerve sheath tumours in individuals with NF1 is unknown but merits further study.

\section{Acknowledgements}

We would like to thank Sofia Granstroem who analysed the neuropsychometric data.

\section{Authors' contributions}

SW contributed to design of the study, collected all 2D MRI morphological data, analyzed all data, and drafted and revised manuscript. VFM contributed to conception and design of the study, recruited patients, played a major role in the acquisition of MRI and neuropsychometric data, and revised the manuscript for intellectual content. RB contributed to the acquisition and analysis of volumetric MRI data, and revised manuscript for intellectual content. SF advised on key statistical analyses and revised the manuscript for intellectual content. PS conducted the volumetric MRI analyses and revised the manuscript for intellectual content. JMF contributed to conception and design of the study and revised the manuscript for intellectual content. MKSH 
contributed to design of the study, guided morphological data collection, and revised the manuscript for intellectual content. JMF and MKSH contributed equally to the manuscript. Allauthors read and approved the manuscript.

\section{Funding}

We are grateful to the Bundesverband Neurofibromatose organization for funding this project.

\section{Availability of data and materials}

Anonymous data are available for appropriate research purposes through V.F. Mautner, MD.

\section{Declarations}

\section{Ethics approval and consent to participate}

The ethical committees of the Medical Chamber in Hamburg and the Research Ethics Board of the University of British Columbia approved the study. Written consent was obtained from all study participants before the study began. All data were de-identified before analysis.

\section{Consent for publication}

Not applicable.

\section{Competing interests}

The authors declare that they have no competing interests.

\section{Author details}

'Department of Medical Genetics, University of British Columbia, Children's and Women's Hospital, 4500 Oak Street, Vancouver, BC V6H 3N1, Canada. ${ }^{2}$ Department of Neurology, University Hospital Hamburg-Eppendorf, Hamburg, Germany. ${ }^{3}$ Department of Nuclear Medicine, University Hospital Hamburg-Eppendorf, Hamburg, Germany. ${ }^{4}$ UBC/LSI Bioinformatics Facility, University of British Columbia, Vancouver, BC, Canada. ${ }^{5}$ Department of Nuclear Medicine, Charité - Universitätsmedizin Berlin, Corporate Member of Freie Universität Berlin, Humboldt-Universität Zu Berlin, and Berlin Institute of Health, Berlin, Germany. ${ }^{6}$ Diagnostic and Therapeutic Neuroradiology, University of British Columbia, Vancouver, Canada.

Received: 12 July 2021 Accepted: 24 October 2021

Published online: 02 November 2021

\section{References}

1. Xu GF, O'Connell P, Viskochil D, Cawthon R, Robertson M, Culver M, et al. The neurofibromatosis type 1 gene encodes a protein related to GAP. Cell. 1990;62(3):599-608.

2. Ballester R, Marchuk D, Boguski M, Saulino A, Letcher R, Wigler M, et al. The NF1 locus encodes a protein functionally related to mammalian GAP and yeast IRA proteins. Cell. 1990;63(4):851-9.

3. Friedman JM. Epidemiology of neurofibromatosis type 1. Am J Med Genet. 1999;89(1):1-6.

4. Friedman JM, et al. Neurofibromatosis 1. In: Adam MP, Ardinger HH, Pagon RA, Wallace SE, Bean LJH, Stephens K, et al., editors. GeneReviews ${ }^{\circledR}$. Seattle: University of Washington; 1993.

5. North K. Neurofibromatosis type 1. Am J Med Genet. 2000;97(2):119-27.

6. Cutting LE, Cooper KL, Koth CW, Mostofsky SH, Kates WR, Denckla MB, et al. Megalencephaly in NF1: predominantly white matter contribution and mitigation by ADHD. Neurology. 2002;59(9):1388-94.

7. Greenwood RS, Tupler LA, Whitt JK, Buu A, Dombeck CB, Harp AG, et al. Brain morphometry, T2-weighted hyperintensities, and IQ in children with neurofibromatosis type 1. Arch Neurol. 2005;62(12):1904-8.

8. Kayl AE, Moore BD 3rd. Behavioral phenotype of neurofibromatosis, type 1. Ment Retard Dev Disabil Res Rev. 2000;6(2):117-24.

9. Cutting LE, Koth CW, Burnette CP, Abrams MT, Kaufmann WE, Denckla MB. Relationship of cognitive functioning, whole brain volumes, and T2-weighted hyperintensities in neurofibromatosis-1. J Child Neurol. 2000;15(3):157-60.
10. Moore BD 3rd, Slopis JM, Jackson EF, De Winter AE, Leeds NE. Brain volume in children with neurofibromatosis type 1: relation to neuropsychological status. Neurology. 2000;54(4):914-20.

11. Moore BD. Potential influences on mathematical difficulties in children and adolescents with neurofibromatosis, type 1. Dev Disabil Res Rev. 2009;15(1):45-51.

12. Rosser TL, Packer RJ. Neurocognitive dysfunction in children with neurofibromatosis type 1. Curr Neurol Neurosci Rep. 2003;3(2):129-36.

13. Pride NA, Korgaonkar MS, Barton B, Payne JM, Vucic S, North KN. The genetic and neuroanatomical basis of social dysfunction: lessons from neurofibromatosis type 1. Hum Brain Mapp. 2014;35(5):2372-82.

14. Koth CW, Cutting LE, Denckla MB. The association of neurofibromatosis type 1 and attention deficit hyperactivity disorder. Child Neuropsychol. 2000;6(3):185-94.

15. Mautner VF, Kluwe L, Thakker SD, Leark RA. Treatment of ADHD in neurofibromatosis type 1. Dev Med Child Neurol. 2002;44(3):164-70.

16. Johnson MR, DeClue JE, Felzmann S, Vass WC, Xu G, White R, et al. Neurofibromin can inhibit Ras-dependent growth by a mechanism independent of its GTPase-accelerating function. Mol Cell Biol. 1994;14(1):641-5.

17. Sellmer L, Farschtschi S, Marangoni M, Heran MK, Birch P, Wenzel R, et al. Non-optic glioma in adults and children with neurofibromatosis 1. Orphanet J Rare Dis. 2017;12(1):34

18. Sellmer L, Farschtschi S, Marangoni M, Heran MKS, Birch P, Wenzel R, et al. Serial MRIs provide novel insight into natural history of optic pathway gliomas in patients with neurofibromatosis 1. Orphanet J Rare Dis. 2018;13(1):62

19. Sevick RJ, Barkovich AJ, Edwards MS, Koch T, Berg B, Lempert T. Evolution of white matter lesions in neurofibromatosis type 1: MR findings. AJR Am J Roentgenol. 1992;159(1):171-5.

20. Steen RG, Taylor JS, Langston JW, Glass JO, Brewer VR, Reddick WE, et al. Prospective evaluation of the brain in asymptomatic children with neurofibromatosis type 1: relationship of macrocephaly to $\mathrm{T} 1$ relaxation changes and structural brain abnormalities. AJNR Am J Neuroradiol. 2001:22(5):810-7.

21. Karlsgodt KH, Rosser T, Lutkenhoff ES, Cannon TD, Silva A, Bearden CE. Alterations in white matter microstructure in neurofibromatosis-1. PLoS ONE. 2012;7(10):e47854.

22. Said SM, Yeh TL, Greenwood RS, Whitt JK, Tupler LA, Krishnan KR. MRI morphometric analysis and neuropsychological function in patients with neurofibromatosis. NeuroReport. 1996;7(12):1941-4.

23. Dubovsky EC, Booth TN, Vezina G, Samango-Sprouse CA, Palmer KM, Brasseux CO. MR imaging of the corpus callosum in pediatric patients with neurofibromatosis type 1. AJNR Am J Neuroradiol. 2001;22(1):190-5.

24. Pride N, Payne JM, Webster R, Shores EA, Rae C, North KN. Corpus callosum morphology and its relationship to cognitive function in neurofibromatosis type 1. J Child Neurol. 2010;25(7):834-41.

25. Wignall EL, Griffiths PD, Papadakis NG, Wilkinson ID, Wallis LI, Bandmann $\mathrm{O}$, et al. Corpus callosum morphology and microstructure assessed using structural MR imaging and diffusion tensor imaging: initial findings in adults with neurofibromatosis type 1. AJNR Am J Neuroradiol. 2010;31(5):856-61.

26. DiMario FJ Jr, Ramsby G, Greenstein R, Langshur S, Dunham B. Neurofibromatosis type 1: magnetic resonance imaging findings. J Child Neurol. 1993:8(1):32-9.

27. Ji J, Shimony J, Gao F, McKinstry RC, Gutmann DH. Optic nerve tortuosity in children with neurofibromatosis type 1. Pediatr Radiol. 2013;43(10):1336-43.

28. Wang S, Friedman JM, Suppa P, Buchert R, Mautner VF. White matter is increased in the brains of adults with Neurofibromatosis 1. medRxiv. 2021.

29. Kayl AE, Moore BD 3rd, Slopis JM, Jackson EF, Leeds NE. Quantitative morphology of the corpus callosum in children with neurofibromatosis and attention-deficit hyperactivity disorder. J Child Neurol. 2000;15(2):90-6.

30. Aydin S, Kurtcan S, Alkan A, Guler S, Filiz M, Yilmaz TF, et al. Relationship between the corpus callosum and neurocognitive disabilities in children with NF-1: diffusion tensor imaging features. Clin Imaging. 2016;40(6):1092-5.

31. Rosset A, Spadola L, Ratib O. OsiriX: an open-source software for navigating in multidimensional DICOM images. J Digit Imaging; 2004. 
32. Riccardi V. The multiple forms of neurofibromatosis. Pediatr Rev. 1982;3:293-8.

33. Riccardi VM. Neurofibromatosis: clinical heterogeneity. Curr Probl Cancer. 1982; $7(2): 1-34$

34. American Academy of Family Physicians, American Psychiatric Association. Work Group on DSM-IV-PC. Diagnostic and statistical manual of mental disorders, fourth edition: primary care version. 1st ed. Washington, DC: American Psychiatric Association; 1995. xv, 223 p.

35. Wechsler D. WAIS-R manual: Wechsler adult intelligence scale-revised. Psychological Corporation; 1981

36. Greenberg LM. The test of variables of attention. 8.0. Los Alamitos: The TOVA Company; 2011.

37. Team R. RStudio: integrated development for R. 3.6.1. Boston: RStudio, Inc.; 2016.

38. Benjamini $Y$, Hochberg $Y$. Controlling the false discovery rate: a practical and powerful approach to multiple testing. J R Stat Soc Ser B (Methodol). 1995;57(1):289-300.

39. van der Knaap MS, Valk J. Myelin and white matter. In: van der Knaap MS, Valk J, editors. Magnetic resonance of myelin, myelination, and myelin disorders. Berlin: Springer; 1995. p. 1-17.

40. Gutmann DH, Parada LF, Silva AJ, Ratner N. Neurofibromatosis type 1: modeling CNS dysfunction. J Neurosci. 2012;32(41):14087-93.

41. Purger D, Gibson EM, Monje M. Myelin plasticity in the central nervous system. Neuropharmacology. 2016;110(Pt B):563-73.

42. Ford AA, Colon-Perez L, Triplett WT, Gullett JM, Mareci TH, Fitzgerald DB. Imaging white matter in human brainstem. Front Hum Neurosci. 2013;7:400.

43. Doraiswamy PM, Figiel GS, Husain MM, McDonald WM, Shah SA, Boyko $\mathrm{OB}$, et al. Aging of the human corpus callosum: magnetic resonance imaging in normal volunteers. J Neuropsychiatry Clin Neurosci. 1991;3(4):392-7.

44. Prendergast DM, Ardekani B, Ikuta T, John M, Peters B, DeRosse P, et al. Age and sex effects on corpus callosum morphology across the lifespan. Hum Brain Mapp. 2015;36(7):2691-702.
45. Igbinedion B, Ogbeide O. Measurement of normal ocular volume by the use of computed tomography. Niger J Clin Pract. 2013;16(3):315-9.

46. Ahmadi H, Shams PN, Davies NP, Joshi N, Kelly MH. Age-related changes in the normal sagittal relationship between globe and orbit. J Plast Reconstr Aesthet Surg. 2007;60(3):246-50.

47. Xie Y, Chen YA, De Bellis MD. The relationship of age, gender, and IQ with the brainstem and thalamus in healthy children and adolescents: a magnetic resonance imaging volumetric study. J Child Neurol. 2012;27(3):325-31.

48. Bai J, Abdul-Rahman MF, Rifkin-Graboi A, Chong YS, Kwek K, Saw SM, et al. Population differences in brain morphology and microstructure among Chinese, Malay, and Indian neonates. PLoS ONE. 2012;7(10):e47816.

49. Isamah N, Faison W, Payne ME, MacFall J, Steffens DC, Beyer JL, et al. Variability in frontotemporal brain structure: the importance of recruitment of African Americans in neuroscience research. PLOS ONE. 2010;5(10):e13642.

50. Kang DW, Wang SM, Na HR, Park SY, Kim NY, Lee CU, et al. Differences in cortical structure between cognitively normal East Asian and Caucasian older adults: a surface-based morphometry study. Sci Rep. 2020:10(1):20905.

51. Trevisson E, Cassina M, Opocher E, Vicenzi V, Lucchetta M, Parrozzani $R$, et al. Natural history of optic pathway gliomas in a cohort of unselected patients affected by Neurofibromatosis 1. J Neurooncol. 2017;134(2):279-87

52. Hyman SL, Shores A, North KN. The nature and frequency of cognitive deficits in children with neurofibromatosis type 1. Neurology. 2005:65(7):1037-44.

\section{Publisher's Note}

Springer Nature remains neutral with regard to jurisdictional claims in published maps and institutional affiliations.
Ready to submit your research? Choose BMC and benefit from:

- fast, convenient online submission

- thorough peer review by experienced researchers in your field

- rapid publication on acceptance

- support for research data, including large and complex data types

- gold Open Access which fosters wider collaboration and increased citations

- maximum visibility for your research: over $100 \mathrm{M}$ website views per year

At $\mathrm{BMC}$, research is always in progress.

Learn more biomedcentral.com/submissions 